\title{
NOTES
}

\section{Thermal Properties of Polyvinylpyridine Solutions}

\author{
Amira Z. DAKRoury, Massarrat B. S. OSMAN, \\ and Abo El-Khair B. MostafA* \\ University College for Women, Ain Shams University, \\ Cairo, Egypt
}

(Received December 7, 1988)

\begin{abstract}
KEY WORDS Poly-2-vinylpyridine / Thermal Conductivity / Thermal Diffusivity / Specific Heat Capacity / Heat Transfer / Conduction /
\end{abstract}

Much work has been reported in the literature concerning investigations of the various properties of polymers and their solutions. ${ }^{1}$ In these investigations, the thermal properties of polymer solutions have received somewhat less attention than many other properties. Since a knowledge of such parameters, i.e., thermal conductivity, thermal diffusivity and specific heat capacity, for polymers in different solutions is of interest both from the point view of industry with its concern for the fabrication and application of materials and also from an academic viewpoint where is an interest in the interrelation of physical properties and structure.

The present work represents an investigation of thermal properties (specific heat capacity $\rho c_{\mathrm{p}}$, thermal diffusivity $a$ and thermal conductivity $\lambda$ ) of poly(2-vinylpyridine) (P2VP) solutions in various solvents at the temperature range $20-90^{\circ} \mathrm{C}$. An experimental setup for the simultaneous absolute measurement of thermal activity $b$, thermal diffusivity $a$ and specific heat capacity $\rho c_{\mathrm{p}}$ of liquids with $\mathrm{AC}$ heated wire (strip) technique is used. ${ }^{2}$ The theory of the plane temperature wave method for determining the thermal activity $b=\lambda /(a)^{1 / 2}$ of the investigated liquid is used combined with the radial heat flow method for determining the thermal diffusivity of a liquid. As a result of this combination all the thermal properties of liquids were determined.

A metallic foil is immersed in the liquid under investigation to produce plane temperature wave. The foil is heated by means of an alternating current with angular frequency $\omega$.

The amplitude of temperature oscillation $\left(\theta_{0}\right)$ of a strip is related to the thermal activity of the investigated liquid according to the followig formula:

$$
b=\frac{d}{2}\left[\frac{1}{2}\left(\frac{W_{0}}{\mathrm{~d} \sqrt{w} S \theta_{0}}\right)^{2}-1\right]^{1 / 2}-1
$$

where $d=\sqrt{w}\left(\rho c_{\mathrm{p}}\right)_{\mathrm{s}} h, W_{0}$ is the power, $S$ is the area of one side of the strip, $\left(\rho c_{\mathrm{p}}\right)_{\mathrm{s}}$ is the heat capacity of the strip material and $h$ is the thickness of the strip.

The amplitude of temperature oscillations of wire $(\theta)$ is related to the thermal properties of the liquid and the wire heat capacity $\left(\rho c_{\mathrm{p}}\right)_{\mathrm{s}}$ according to the following relation:

$$
\theta=\theta_{0}\left(\frac{\operatorname{her}^{2}(X)+\operatorname{hei}^{2}(X)}{\left[X \eta \operatorname{hei}(X)+\operatorname{her}^{\prime}(X)\right]^{2}+\left[X \eta \operatorname{her}(X)-\operatorname{hei}^{\prime}(X)\right]^{2}}\right)^{1 / 2}
$$


where $X=\sqrt{(2 w / a)} r, \eta=\left(\rho c_{\mathrm{p}}\right)_{\mathrm{s}} /\left(2 \rho c_{\mathrm{p}}\right)_{1}, r$ is the radius of the wire, $\rho$ is the density, $\theta_{0}$ is the amplitude of the temperature oscillations of a noninertial strip and her, hei, her', hei' are Hankel functions and derivatives. From eq 1 and $2, a, \lambda$ and $\rho c_{\mathrm{p}}$ of liquid or solution under investigation can be calculated. This technique proved to much suitable since the liquid layer around the sensor is thin enough to suppress the hydrodynamic current leading to the elimination of the convective heat transport. ${ }^{3}$

The calculated systematic errors of the thermal diffusivity and heat capacity coefficients are not more than $2.5 \%$. Maximum errors for the thermal conductivity measurements were $2.2 \%$.

The measurements were carried out in: ethyl alcohol, acetic acid, $N, N$-dimethylformamide (DMF) and benzene with various concentrations of poly-2-vinylpyridine (from $0.3 \%$ up to $1.0 \%$ ). The molecular weight of P-2VP was estimated to be $2.85 \times 10^{5}$ from the intrinsic viscosity in DMF at $25^{\circ} \mathrm{C}{ }^{1}$

Poly-2-vinylpyridine is an important compound and has been used for a variety of functions as a good inhibitor for aqueous corrosion of metals as $\mathrm{Al}, \mathrm{Fe}$, and $\mathrm{Zn} .^{4,5}$

The results of specific heat capacity for P2VP using different solvents: ethyl alcohol, acetic acid, DMF and benzene, in the temperature range $20-90^{\circ} \mathrm{C}$ indicate that, it increases as the temperature rises up. For P-2VP solution in benzene as a solvent, $\rho c_{\mathrm{p}}$ decreases with increasing the concentration of P-2VP in the medium. However, for the other solvents, it increases as the concentration of P-2VP increases.

The results of thermal diffusivity of P-2VP in different solvents and temperatures show that, it decreases as the concentration of $\mathrm{P}$ $2 \mathrm{VP}$ increases. In addition, the values of such parameter for P-2VP solutions depend on the type of the used solvent in the system.

The thermal conductivity measurements for $\mathrm{P}-2 \mathrm{VP}$ in different solvents at the temperature range $20-90^{\circ} \mathrm{C}$ are illustrated in Figure $1(\mathrm{a}-$ d). In general, it is observed that the values of thermal conductivity decreases with the addition of P-2VP in the solvent. P-2VP solutions are more conducting in the following order:

ethyl alcohol $>$ acetic acid $>$ dimethylformamide $>$ benzene

Also, the thermal conductivity of P-2VP solutions increases as the temperatures rises. These results indicate that the type of solvent, i.e., polymer-solvent interaction, play an important role on the thermal properties behaviour of the investigated system. Previously, the nature of the specific interaction between P-2VP and various types of solvents was reported by Arichi et al. ${ }^{6}$ The nitrogen atom at the $\alpha$-position of the pyridine ring of P-2VP has a lone electron pair and acts as an electron donor, and so P-2VP is quite a strong base. When a solvent is a Lewis acid or can act as an electron acceptor, its solvent power with regard to the polymer may be increased because of solvation. ${ }^{6}$ This confirms the obtained results, where higher conduction for P-2VP solutions was observed in ethyl alcohol and acetic acid than in the other solvents.

In absence of P-2VP, the differences in the thermal conductivity parameter for the investigated liquids mainly depend on their nature. Thus, the polarity of the investigated solvents are different and it increases as follows:

dimethylformamide $>$ ethyl alcohol $>$ acetic acid $>$ benzene

Since the dielectric constants of DMF, ethyl alcohol, acetic acid, and benzene are 38.00, $24.30,6.15$, and 2.27 , respectively. ${ }^{7}$ Also, ethyl alcohol and acetic acid are protic solvents, while DMF is dipolar aprotic solvent. It is clear that, the thermal conduction which is responsible for the mechanism of heat transfer ${ }^{3}$ for polar protic solvents (ethyl alcohol and acetic acid) are higher than that of polar aprotic (DMF) or weakly polar aprotic (benzene) ones. In polymeric solutions, P-2VP 


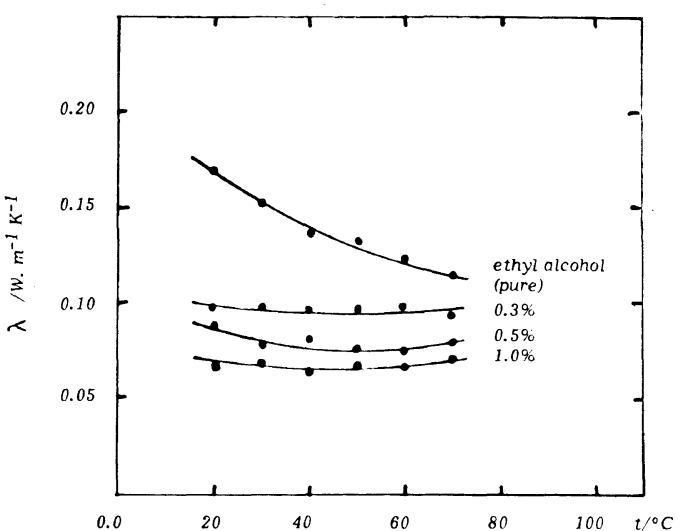

(a)

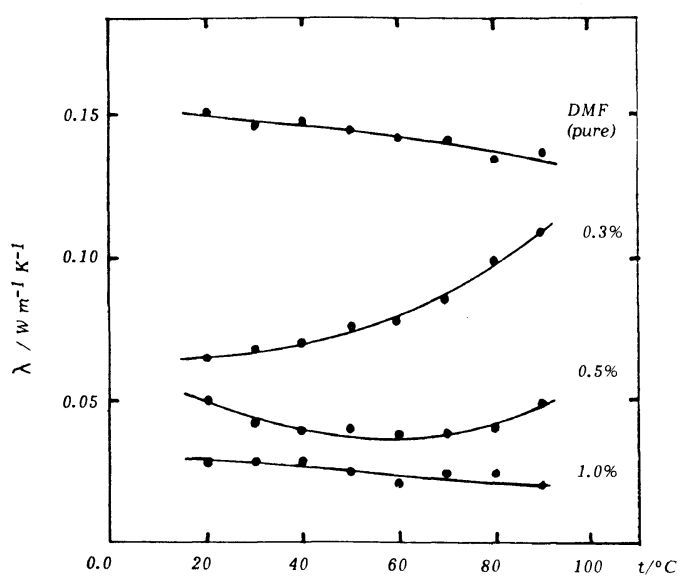

(c)

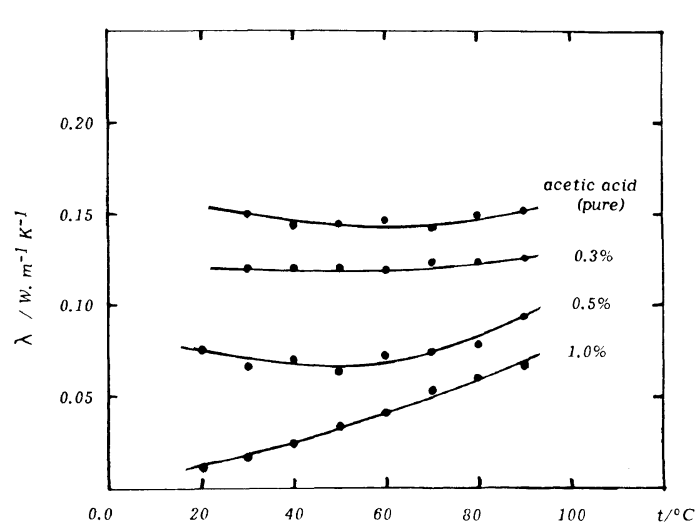

(b)

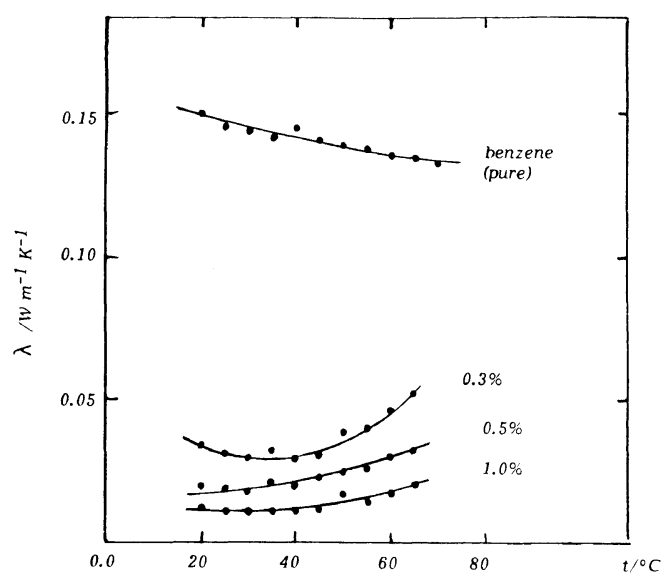

(d)

Figure 1. Variation of thermal conductivity with temperature for various concentrations of poly-2vinylpyridine in different solvents: a) ethyl alcohol; b) acetic acid; c) dimethylformamide; d) benzene.

changes the nature of the solvents during their solvation with its molecular chains leading to a decrease in the values of $\lambda$. Nevertheless, as the temperature rises, the order of solvation decreases which influences the thermal conductivity parameter by increasing it.

Figure $1(\mathrm{a}-\mathrm{d})$ also show that the thermal conductivity decreases as the concentration of $\mathrm{P}-2 \mathrm{VP}$ increases in the system. The decrease in $\lambda$ with concentration is related to the decrease of the mean free path of poly-2-vinylpyridine molecules in solution.

From the above results it can be concluded that the mechanism of heat transfer is due to conduction and the obtained values of thermal properties depend mainly on (i) the type of solvent, (ii) the concentrations of $\mathrm{P}-2 \mathrm{VP}$ in the system, and (iii) the temperatures.

\section{REFERENCES}

1. J. Brandrup and E. H. Immergut, "Polymer Handbook," 2nd ed, Wiley-Interscience, New York, N. Y., 1966, pp VI-2, VI-18.

2. S. R. Atalla, A. A. El-Sharkawy, and F. A. Gasser, Int. Thermophys. J., 2, 155 (1981).

3. A. A. El-Sharkawy, M. A. Kenawy, and A. Z. 
Dakroury, High Temperature-High Pressures, 15, 391 (1983).

4. B. M. Abo El-Khair, S. M. Abdel-Wahab, and E. M. Mabrouk, Surface and Coatings Technology, 27, 317 (1986).

5. B. M. Abo El-Khair, I. A. Abdel-Hamid, and O. R. Khalifa, 31st International Congress of Pure and
Applied Chemistry (IUPAC), Sofia, Bulgaria, July 1987, pp 13-18.

6. S. Arichi, H. Matsuura, Y. Tanimoto, and $\mathrm{H}$. Murata, Bull. Chem. Soc. Jpn., 39, 434 (1966).

7. "Lange's Handbook of Chemistry," McGraw-Hill, Inc., New York, N. Y., 1973, pp 10-148. 